www.nature.com $/ \mathrm{hr}$

\title{
Significance of measuring oxidative stress in lifestyle-related diseases from the viewpoint of correlation between d-ROMs and BAP in Japanese subjects
}

\begin{abstract}
Toshiki Fukui ${ }^{1}$, Kazuhiro Yamauchi ${ }^{1}$, Mie Maruyama ${ }^{1}$, Tadashi Yasuda ${ }^{1}$, Masakazu Kohno ${ }^{2}$ and Youichi Abe ${ }^{1}$
In recent years, oxidative stress has been postulated to be an important factor in the pathogenesis and development of lifestyle-related diseases. In this study, we investigated the association between the derivatives of reactive oxygen metabolites (d-ROMs), as an index of products of reactive oxygen species (ROS), and biological antioxidant potential (BAP), as an index of antioxidant potential. We also investigated the associations between d-ROMs or BAP and the risk factors for lifestyle-related diseases or metabolic syndrome-associated factors to evaluate their usefulness in preventive medicine. There were 442 subjects who underwent health checkup examination in our facilities. In addition to standard medical checkup items, we analyzed d-ROMs, BAP, brachial-ankle pulse wave velocity, high-sensitivity C-reactive protein level and visceral fat area (VFA) visualized on a computed tomography scan. The mean d-ROM value in females was significantly higher than that in males. There was a positive correlation between the d-ROM and VFA levels. On correlation analysis, there was a negative correlation between the d-ROM and creatinine levels. As factors that influence d-ROMs, the level of VFA was selected, suggesting the significance of oxidative stress measurement with d-ROMs. In addition, there was a positive correlation between d-ROMs and BAP values. Further research is required to resolve whether increased production of ROS or the antioxidant potential that can compensate for such an increase of ROS is more important in vivo.
\end{abstract} Hypertension Research (2011) 34, 1041-1045; doi:10.1038/hr.2011.76; published online 16 June 2011

Keywords: BAP; C-reactive protein; d-ROMs; oxidative stress; visceral fat

\section{INTRODUCTION}

Oxidative stress may be involved in various disorders and pathogeneses, especially in lifestyle-related diseases, and it causes the progression and acceleration of atherosclerosis. ${ }^{1-3}$ Biomarkers of oxidative stress, such as serum malondialdehyde level measured as thiobarbituric acid-reactive substances, oxidized low-density lipoprotein, oxidation injury of DNA, 8-hydroxydeoxyguanosine and urinary excretion of 8 -iso-prostaglandin-F2 $\alpha$, are generally measured in laboratories. ${ }^{4-7}$ On the other hand, as indices of antioxidant potential, intracellular levels of superoxide dismutase and glutathione peroxidase have mainly been measured. ${ }^{8-9}$

However, these assay methods are complex and are not suitable for analysis of a large number of subjects. Recently, simpler methods for detecting reactive oxygen species (ROS) using derivatives of reactive oxygen metabolites (d-ROMs) and biological antioxidant potential (BAP) were developed, and reports on studies using these methods have been increasing. ${ }^{10-20}$ Thus, in the present study, we measured dROMS and BAP as indices for the production of ROS and antioxidant potential, respectively.
As oxidative stress has been explained to show the balance of production of ROS and the capacity of antioxidant, it is more appropriate that not only the production of ROS but also antioxidant capacity be evaluated at the same time. Both d-ROM and BAP levels can be measured simultaneously in large numbers of samples using an all-purpose automatic analyzer. ${ }^{10}$ Therefore, we measured d-ROM and BAP levels in the same subjects to evaluate their association.

\section{METHODS}

Study subjects

The subjects were 442 individuals who underwent a health checkup examination in our facilities ( 358 males and 84 females). Informed consent was given by all subjects before the study.

\section{Laboratory measurements}

Data on lifestyle-related factors were recorded, and measurements on serum high-sensitivity C-reactive protein (hsCRP) and creatinine were performed using an automatic analyzer (Hitachi, Ibaraki, Japan).

${ }^{1}$ Center for Preventive Medical Treatment, NTT West Takamatsu Hospital, Takamatsu, Japan and ${ }^{2}$ Department of Cardiorenal and Cerebrovascular Internal Medicine, Kagawa University Medical School, Takamatsu, Japan

Correspondence: Dr T Fukui, Center for Preventive Medical Treatment, NTT West Takamatsu Hospital, Takamatsu, Japan.

E-mail: ftoshi@kagawa.west.ntt.co.jp

Received 15 November 2010; revised 16 March 2011; accepted 31 March 2011; published online 16 June 2011 


\section{Assay of oxidative stress and antioxidant potential}

We used a simple method to detect hydroperoxide levels by measuring d-ROMs. Hydroperoxides are converted into radicals that oxidize $\mathrm{N}, \mathrm{N}$-diethyl-para-phenylenediamine and can be detected spectrophotometrically using an all-purpose automatic analyzer. This can be measured simultaneously with other inspection items. The results of the d-ROM level are expressed in an arbitrary unit called Carratelli unit (U.CARR). ${ }^{10-12}$

The BAP level was measured as antioxidant potential and also measured using an all-purpose automatic analyzer. This test examines the blood concentration of antioxidants as agents that can reduce iron from ferric $\left(\mathrm{Fe}^{3+}\right)$ to ferrous form $\left(\mathrm{Fe}^{2+}\right)$. We estimated the intensity of this chromatic change photometrically. The results are expressed as $\mu \mathrm{moll}^{-1}{ }^{10}$

We performed precision tests in our laboratory to evaluate the reproducibility and accuracy of the method for measuring d-ROMs and BAP. Upon consecutively measuring samples 10 times, the coefficient of variation ranged from 0.2 to $2.1 \%$ for d-ROMs and from 0.1 to $1.1 \%$ for BAP. When performing measurement for 8 days, the inter-measurement coefficient of variation values were $1.0-1.1 \%$ and $3.0-3.2 \%$, respectively.

Results from these two tests showed a normal distribution of values.

\section{Brachial-ankle pulse wave velocity measurements}

The brachial-ankle pulse wave velocity of each subject was measured using a non-invasive automatic oscillometric device (PWV/ABI, BP-203RPE; Omron Colin, Tokyo, Japan) in a supine position after $5 \mathrm{~min}$ of bed rest as described previously. ${ }^{21}$

\section{Measuring fat distribution}

Abdominal fat distribution was determined using computed tomography while the subjects were in a supine position. The subcutaneous fat area and intraabdominal visceral fat area (VFA) were measured at the level of the umbilicus and determined by a standardized method with computed tomography numbers.

\section{Statistical analysis}

Continuous data are expressed as the means \pm s.d. Comparison of continuous variables among the three groups was performed by one-way analysis of variance, with post hoc comparison with Scheffe's method. The linearity of the correlation between two variables was assessed by linear regression. Multivariate analysis was performed using stepwise linear regression.

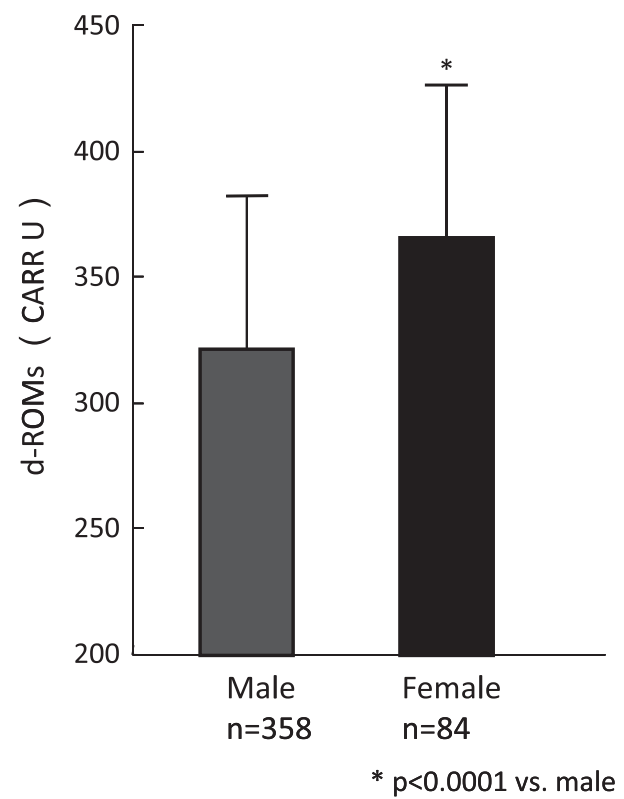

Figure 1 Comparison of derivatives of reactive oxygen metabolites ( $d$-ROMs) between males and females. ${ }^{*} P<0.0001$ vs. male.
A $P$-value $<0.05$ was considered significant. Analyses were conducted using Stat View 5.0 (SAS Institute, Cary, NC, USA).

\section{RESULTS}

The mean age of subjects was $47 \pm 11$ years and $19 \%$ of them were female. The mean d-ROM value in females $(n=84)$ was significantly higher than that in males $(n=358 ; 365 \pm 74$ and $321 \pm 61$ U.CARR, respectively; Figure 1). There was no significant difference according to age.

In multivariate analysis, gender, hsCRP level and VFA were selected as factors that influence d-ROMs (Table 1). However, when excluding data on VFA from the multivariate analysis, the creatinine level was selected as a negative factor; d-ROM-influencing factors slightly changed depending on the factors employed for multivariate analysis. Baseline characteristics of factors employed for multivariate analysis and relationships with d-ROMs are shown in Table 2.

Table 1 Stepwise multiple linear regression analysis with d-ROMs as the dependent variable

\begin{tabular}{lcr}
\hline Variables & $\begin{array}{c}\text { Standardized } \\
\text { regression coefficient }\end{array}$ & F value \\
\hline Gender & 0.288 & 20.871 \\
hsCRP & 0.208 & 10.847 \\
VFA & 0.165 & 6.241
\end{tabular}

Abbreviations: d-ROM, derivatives of reactive oxygen metabolite; hSCRP, high-sensitivity Creactive protein; VFA, visceral fat area.

Adjusted $R^{2}=0.119, P<0.0001$

Table 2 Baseline characteristics of factors employed for multivariate analysis and relationships with d-ROMs

\begin{tabular}{|c|c|c|c|}
\hline Variables & Average & $r$ & P-value \\
\hline Male/female & $358 / 84$ & 0.162 & 0.004 \\
\hline Age (years) & $47 \pm 11$ & 0.121 & 0.034 \\
\hline Waist circumference $(\mathrm{cm})$ & $85 \pm 10$ & 0.105 & 0.066 \\
\hline Total fat area $\left(\mathrm{cm}^{2}\right)$ & $198.1 \pm 96.0$ & 0.143 & 0.012 \\
\hline SFA $\left(\mathrm{cm}^{2}\right)$ & $129.8 \pm 68.5$ & 0.105 & 0.066 \\
\hline VFA $\left(\mathrm{cm}^{2}\right)$ & $68.3 \pm 41.8$ & 0.156 & 0.006 \\
\hline $\mathrm{BMI}\left(\mathrm{kg} \mathrm{m}^{-2}\right)$ & $23.7 \pm 3.7$ & 0.057 & 0.322 \\
\hline Systolic blood pressure $(\mathrm{mm} \mathrm{Hg})$ & $123 \pm 16$ & 0.089 & 0.122 \\
\hline Diastolic blood pressure $(\mathrm{mm} \mathrm{Hg})$ & $77 \pm 12$ & -0.002 & 0.966 \\
\hline $\mathrm{HDL}\left(\mathrm{mg} \mathrm{dl}^{-1}\right)$ & $59 \pm 14$ & -0.056 & 0.325 \\
\hline Triglyceride $\left(\mathrm{mg} \mathrm{dl} \mathrm{l}^{-1}\right)$ & $121 \pm 84$ & 0.006 & 0.911 \\
\hline $\operatorname{LDL}\left(\mathrm{mg} \mathrm{dl}^{-1}\right)$ & $118 \pm 31$ & 0.063 & 0.272 \\
\hline Fasting glucose (mg dl ${ }^{-1}$ ) & $97 \pm 14$ & 0.043 & 0.457 \\
\hline $\mathrm{HbAlc}(\%)$ & $5.3 \pm 0.8$ & 0.100 & 0.078 \\
\hline hsCRP (mg dl-1) & $0.09 \pm 0.13$ & 0.194 & $<0.001$ \\
\hline baPWV $\left(\mathrm{cm} \mathrm{s}^{-1}\right)$ & $1359 \pm 251$ & 0.069 & 0.263 \\
\hline Alb (mg dl-1) & $4.7 \pm 0.3$ & 0.049 & 0.431 \\
\hline AST (mg dl-1) & $26 \pm 12$ & 0.155 & 0.010 \\
\hline ALT (mg dl-1) & $31 \pm 20$ & 0.077 & 0.207 \\
\hline$\gamma$-GTP $\left(\mathrm{mg} \mathrm{dl}^{-1}\right)$ & $52 \pm 73$ & 0.043 & 0.483 \\
\hline $\mathrm{UA}\left(\mathrm{mg} \mathrm{dl}^{-1}\right)$ & $5.8 \pm 1.4$ & -0.043 & 0.475 \\
\hline Creatinine $\left(\mathrm{mg} \mathrm{dl}^{-1}\right)$ & $0.83 \pm 0.15$ & -0.208 & $<0.001$ \\
\hline White blood cells $\left(\mathrm{nl}^{-1}\right)$ & $5.7 \pm 1.6$ & 0.179 & 0.003 \\
\hline Current smokers (\%) & 27.8 & & \\
\hline Hypertension (\%) & 12.0 & & \\
\hline Diabetes (\%) & 3.1 & & \\
\hline Dyslipidemia (\%) & 5.2 & & \\
\hline
\end{tabular}

Abbreviations: Alb, albumin; ALT, alanine transaminase; AST, aspartate aminotransferase; baPWV, brachial-ankle pulse wave velocity; BMI, body mass index; d-ROM, derivatives of reactive oxygen metabolite; GTP, guanosine triphosphate; HbAlc, glycohemoglobin A1c; HDL, high-density lipoprotein; hSCRP, high-sensitivity C-reactive protein; LDL, low-density lipoprotein; SFA, subcutaneous fat area; UA, uric acid; VFA, visceral fat area. Values are mean \pm s.d. 
The value of hsCRP was classified into three grades: $<0.10$ (group A), 0.10 to $<0.30$ (group B) and 0.30 to $<0.10$ (group C). The mean values of d-ROMs were $321 \pm 63 \mathrm{U}$.CARR $(n=347)$ in group A,

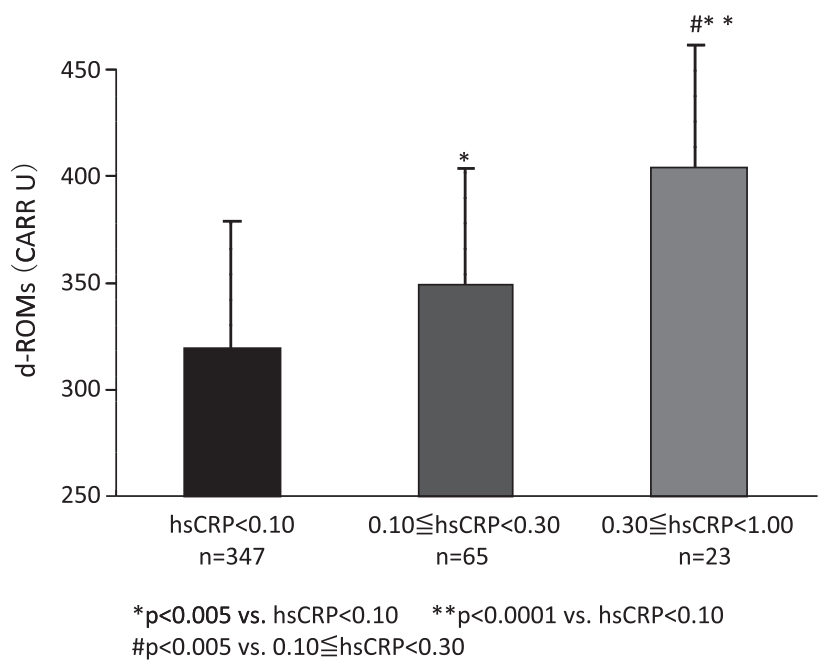

Figure 2 Changes of derivatives of reactive oxygen metabolites (d-ROMs) with high-sensitivity C-reactive protein (hsCRP) level. ${ }^{*} P<0.005$ vs. hsCRP $<0.10$; ${ }^{* *} P<0.0001$ vs. hsCRP $<0.10$ and ${ }^{\#} P<0.005$ vs. $0.10 \leqq$ hsCRP $<0.30$.
$349 \pm 54$ U.CARR $(n=65)$ in group B and $402 \pm 61$ U.CARR $(n=23)$ in group $\mathrm{C}(P<0.0001$ by one-way analysis of variance $)$ and varied among the groups (Figure 2).

There was a positive correlation between d-ROM level and VFA $(n=308, r=0.156, P<0.01)$. However, the former was not correlated with subcutaneous fat area $(n=308, r=0.105, P=0.066$; Figure 3$)$.

On correlation analysis, there was a negative correlation between $\mathrm{d}-\mathrm{ROM}$ and serum creatinine levels $(n=384, r=-0.285, P<0.0001$; Figure $4 \mathrm{a})$. The values of serum creatinine in males $(n=316)$ were classified into three grades: $<0.80$ (group A), 0.80 to $<1.00$ (group B) and $>1.00$ (group $\mathrm{C}$ ). The mean values of $\mathrm{d}$-ROMs were $335 \pm 61$ U.CARR $(n=96)$ in group A, $321 \pm 58$ U.CARR $(n=181)$ in group $\mathrm{B}$ and $308 \pm 62 \mathrm{U}$.CARR $(n=42)$ in group $\mathrm{C}(P<0.05$ by one-way analysis of variance) and there was a significant difference between groups A and C $(P<0.05$; Figure $4 \mathrm{~b})$.

The mean BAP value was $2829 \pm 247 \mu \mathrm{M}(n=229)$. There was no gender or age difference in BAP. As factors that influence BAP, the levels of serum albumin and uric acid were selected for multivariate analysis (Table 3). Baseline characteristics of factors employed for multivariate analysis and relationships with BAP are shown in Table 4.

There was a positive correlation between d-ROM and BAP values ( $n=229, r=0.184, P<0.01$; Figure 5$)$. There was no positive correlation between d-ROMs or BAP and brachial-ankle pulse wave velocity (data not shown).
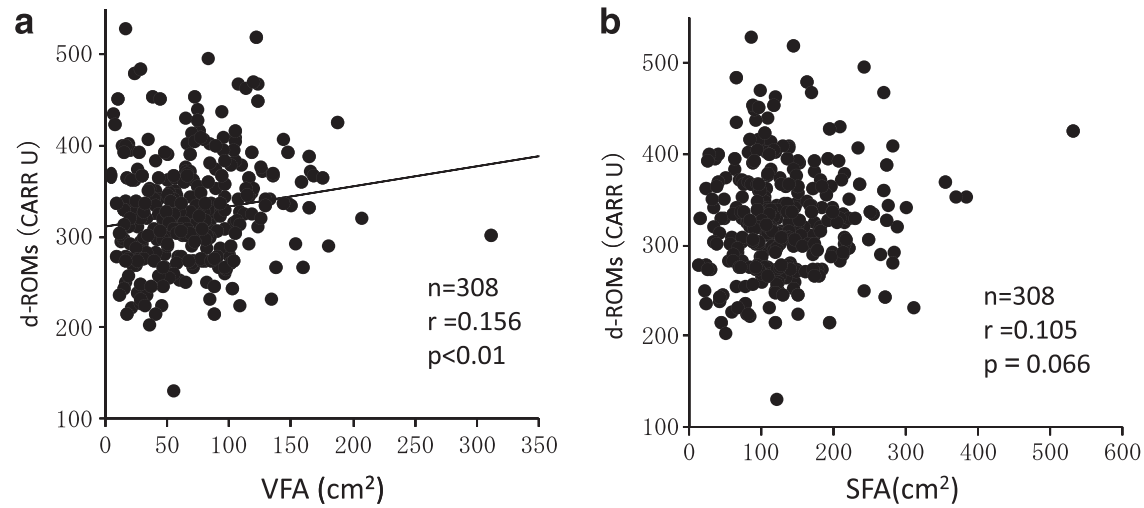

Figure 3 (a) Relationship between derivatives of reactive oxygen metabolites (d-ROMs) and visceral fat area (VFA). (b) Relationship between d-ROMs and subcutaneous fat area (SFA).
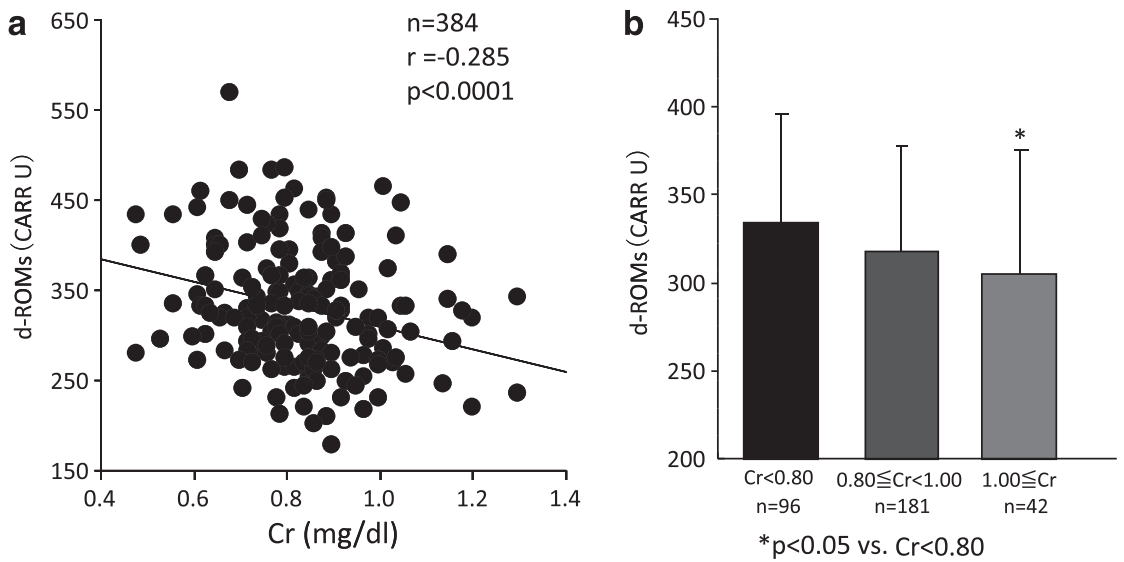

Figure 4 Relationship between derivatives of reactive oxygen metabolites (d-ROMs) and serum creatinine (Cr) level. (a) Correlation between d-ROMs and serum creatinine level. (b) Changes of $\mathrm{d}-\mathrm{ROMs}$ with serum creatinine level in males. ${ }^{*} P<0.05$ vs. $\mathrm{Cr}<0.80$. 
Table 3 Stepwise multiple linear regression analysis with BAP as the dependent variable

\begin{tabular}{lcc}
\hline Variables & $\begin{array}{c}\text { Standardized } \\
\text { regression coefficient }\end{array}$ & F value \\
\hline Alb & 0.255 & 8.407 \\
UA & 0.240 & 7.411 \\
\hline
\end{tabular}

Abbreviations: Alb, albumin; BAP, biological antioxidant potential; UA, uric acid. Adjusted $R^{2}=0.121, P<0.001$

Table 4 Baseline characteristics of factors employed for multivariate analysis and relationships with BAP

\begin{tabular}{|c|c|c|c|}
\hline Variables & Average & r & P-value \\
\hline Male/female & $194 / 35$ & -0.011 & 0.897 \\
\hline Age (years) & $48 \pm 10$ & 0.053 & 0.535 \\
\hline Waist circumference (cm) & $86 \pm 10$ & -0.099 & 0.245 \\
\hline Total fat area $\left(\mathrm{cm}^{2}\right)$ & $206.4 \pm 98.0$ & -0.127 & 0.133 \\
\hline $\mathrm{SFA}\left(\mathrm{cm}^{2}\right)$ & $134.7 \pm 71.7$ & -0.116 & 0.172 \\
\hline VFA $\left(\mathrm{cm}^{2}\right)$ & $71.7 \pm 40.6$ & -0.104 & 0.221 \\
\hline $\mathrm{BMI}\left(\mathrm{kg} \mathrm{m}^{-2}\right)$ & $24.1 \pm 3.7$ & -0.133 & 0.116 \\
\hline Systolic blood pressure (mm Hg) & $122 \pm 16$ & 0.031 & 0.719 \\
\hline Diastolic blood pressure $(\mathrm{mm} \mathrm{Hg})$ & $76 \pm 12$ & -0.007 & 0.932 \\
\hline $\mathrm{HDL}\left(\mathrm{mg} \mathrm{dl}{ }^{-1}\right)$ & $60 \pm 15$ & -0.011 & 0.899 \\
\hline Triglyceride (mg dl ${ }^{-1}$ ) & $122 \pm 74$ & -0.027 & 0.754 \\
\hline LDL (mg dl-1) & $121 \pm 28$ & 0.009 & 0.920 \\
\hline Fasting glucose (mg dl ${ }^{-1}$ ) & $96 \pm 14$ & 0.015 & 0.859 \\
\hline $\mathrm{HbA} 1 \mathrm{c}(\%)$ & $5.3 \pm 0.8$ & 0.045 & 0.600 \\
\hline hsCRP (mg dl-1) & $0.08 \pm 0.21$ & 0.098 & 0.267 \\
\hline baPWV $\left(\mathrm{cm} \mathrm{s}^{-1}\right)$ & $1383 \pm 272$ & 0.029 & 0.738 \\
\hline Alb (mg dl $\left.{ }^{-1}\right)$ & $4.7 \pm 0.3$ & 0.243 & 0.004 \\
\hline AST (mg dl-1) & $23 \pm 10$ & -0.046 & 0.585 \\
\hline $\operatorname{ALT}\left(\mathrm{mg} \mathrm{dl}^{-1}\right)$ & $31 \pm 23$ & 0.055 & 0.516 \\
\hline$\gamma$-GTP $\left(\mathrm{mg} \mathrm{dl}^{-1}\right)$ & $62 \pm 92$ & 0.052 & 0.544 \\
\hline $\mathrm{UA}\left(\mathrm{mg} \mathrm{dl}^{-1}\right)$ & $5.9 \pm 1.4$ & 0.232 & 0.006 \\
\hline Creatinine (mg dl-1) & $0.83 \pm 0.14$ & 0.108 & 0.200 \\
\hline White blood cells $\left(\mathrm{nl}^{-1}\right)$ & $5.8 \pm 1.6$ & 0.090 & 0.297 \\
\hline Current smokers (\%) & 30.1 & & \\
\hline Hypertension (\%) & 11.2 & & \\
\hline Diabetes (\%) & 1.3 & & \\
\hline Dyslipidemia (\%) & 4.6 & & \\
\hline
\end{tabular}

Abbreviations: Alb, albumin; ALT, alanine transaminase; AST, aspartate aminotransferase; BAP, biological antioxidant potential; baPWV, brachial-ankle pulse wave velocity; BMI, body mass index; GTP, guanosine triphosphate; $\mathrm{HbAlc}$, glycohemoglobin Alc; HDL, high-density lipoprotein; hsCRP, high-sensitivity C-reactive protein; LDL, low-density lipoprotein; SFA subcutaneous fat area; UA, uric acid; VFA, visceral fat area. Subcutaneous fat area;
Values are mean \pm s.d.

\section{DISCUSSION}

Oxidative stress may be involved in various disorders and pathogeneses. Many studies have shown its involvement in the pathogeneses of all lifestyle-related diseases. Previous human studies have employed markers of ROS, such as 8-hydroxydeoxyguanosine and 8-iso-prostaglandin-F2 $\alpha$. However, it is difficult to measure them at health checkup facilities. Furthermore, as an index of the antioxidant potential, the level of superoxide dismutase was also difficult to measure, even at research facilities.

In this study, we used a simpler method and investigated the association of d-ROMs, as an index of ROS production, and BAP, as an index of antioxidant potential, at the same time. Recent studies have shown the usefulness of the evaluation of oxidative stress using

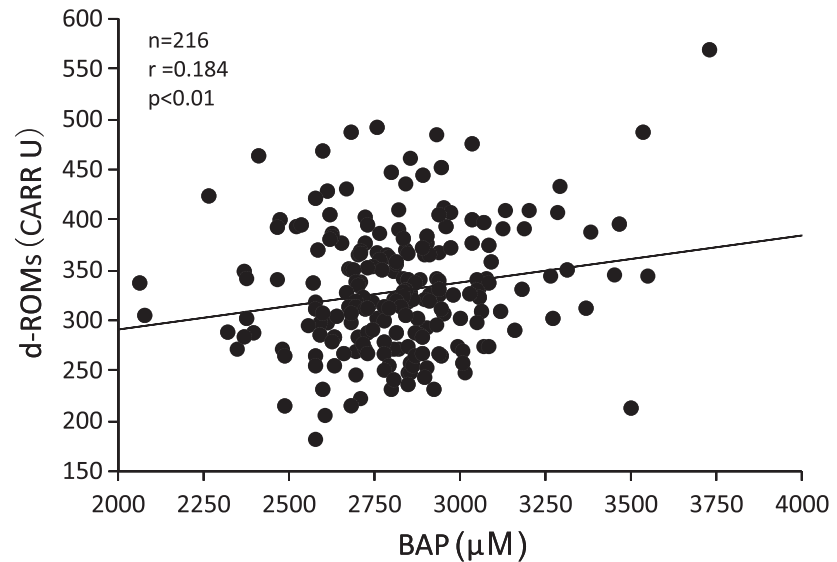

Figure 5 Relationship between derivatives of reactive oxygen metabolites (d-ROMs) and biological antioxidant potential (BAP).

d-ROMs. ${ }^{13-16}$ However, this is the first study to measure them simultaneously in a large number of subjects to evaluate oxidative stress from the viewpoint of lifestyle-related disease.

Trotti et al. ${ }^{12}$ reported that no statistically significant difference was found between genders in European subjects. However, the mean $\mathrm{d}$-ROM value in females was significantly higher than that in males in Japanese subjects. In multivariate analysis, gender, hsCRP level and VFA were selected as factors that influence d-ROMs. Recent studies have also shown the positive correlation between the levels of hsCRP and d-ROMs. ${ }^{14-16}$

Chronic inflammatory processes have been recognized to have a role in the pathogenesis of atherosclerosis. ${ }^{22-24}$ Therefore, an increase of visceral fat tissue that promotes inflammation, as represented by hsCRP, may have a role in the pathogenesis of atherosclerosis; our result suggests the significance of measuring d-ROMs. Recently, Fujita et $a .^{25}$ reported that oxidative stress is associated with visceral fat accumulation.

On the other hand, d-ROM level significantly decreased with an increase in the level of creatinine. However, as this may contribute to the gender difference in d-ROM level, this must be considered when interpreting values in elderly subjects and those with renal hypofunction.

As factors that influence BAP, the levels of serum albumin and uric acid were selected. Both are recognized as the primary defense against oxidative stress in extracellular fluids. ${ }^{26}$ This suggests that the result of BAP measurement reflects the antioxidant capacity.

Oxidative stress may be associated with not only lifestyle-related diseases but also various other diseases. Therefore, our results suggest the significance of oxidative stress measurement in a large number of subjects, although data with a relatively weak correlation are included in part of our research results.

Humans have the longest lifespan among mammalian species, and it has been shown that lifespan is correlated with the level of superoxide dismutase activity. ${ }^{27}$ The mean BAP in Japanese subjects was markedly higher than that previously reported in European/American subjects. ${ }^{28}$ This may be one cause of the longevity of the Japanese. However, further epidemiological studies should be carried out to support this hypothesis.

Oxidative stress is postulated to be a major causal factor of aging. However, it is still not clear which factor is more critical for an increase in reactive oxygen and/or decrease in antioxidant defense. 
The lifespan of females is usually longer than that of males. However, the level of d-ROMs in females was higher than that in males in our study. Furthermore, there was a positive correlation between the levels of d-ROMs and BAP. These results indicate that further research will be required to resolve which is more important in vivo, the increased production of ROS or the antioxidant potential that can compensate for such an increase of ROS.

Recently, many results of evaluations of oxidative stress using d-ROMs and BAP have been reported. However, there are only a few papers that show the relationship between these parameters and other conventional indices of oxidative stress directly. ${ }^{17-18}$ Lubrano et al. ${ }^{17}$ showed a good correlation between d-ROMs and 8-iso-prostaglandinF2 $\alpha$. However, their accuracy must be analyzed more in the future.

In conclusion, this is the first study reporting that not only hsCRP but also VFA is positively correlated with the level of d-ROMs. Furthermore, this is the first study to measure d-ROMs and BAP in the same subjects to evaluate oxidative stress from the viewpoint of lifestyle-related disease.

\section{CONFLICT OF INTEREST}

The authors declare no conflict of interest.

\section{ACKNOWLEDGEMENTS}

We are grateful to Dr Iorio EL for excellent technical assistance in writing the paper.

1 Aviram M. Review of human studies on oxidative damage and antioxidant protection related to cardiovascular diseases. Free Radic Res 2000; 33: S85-S97.

2 Griendling KK, FitzGerald GA. Oxidative stress and cardiovascular injury: Part II: animal and human studies. Circulation 2003; 108: 2034-2040.

3 Harrison D, Griendling KK, Landmesser U, Hornig B, Drexler H. Role of oxidative stress in atherosclerosis. Am J Cardiol 2003; 91: 7A-11A.

4 Walter MF, Jacob RF, Jeffers B, Ghadanfar MM, Preston GM, Buch J, Mason RP. Serum levels of thiobarbituric acid reactive substances predict cardiovascular events in patients with stable coronary artery disease: a longitudinal analysis of the PREVENT study. J Am Coll Cardiol 2004; 44: 1996-2002.

5 Shimada K, Mokuno H, Matsunaga E, Miyazaki T, Sumiyoshi K, Miyauchi K, Daida H. Circulating oxidized low-density lipoprotein is an independent predictor for cardiac event in patients with coronary artery disease. Atherosclerosis 2004; 174: 343-347.

6 Wu LL, Chiou CC, Chang PY, Wu JT. Urinary 8-OHdG A marker of oxidative stress to DNA and a risk factor for cancer, atherosclerosis and diabetics. Clin Chim Acta 2004 339: 1-9.

7 Schwedhelm E, Bartling A, Lenzen H, Tsikas D, Maas R, Brummer J, Gutzki FM, Berger $\mathrm{J}$, Frölich JC, Boger RH. Urinary 8-iso-prostaglandin F2 alpha as a risk marker in patients with coronary heart disease: a matched case-control study. Circulation 2004, 109. $843-848$.
8 Faraci FM, Didion SP. Vascular protection: superoxide dismutase isoforms in the vessel wall. Arterioscler Thromb Vasc Biol 2004; 24: 1367-1373.

9 Pyne-Geithman GJ, Caudell DN, Prakash P, Clark JF. Glutathione peroxidase and subarachnoid hemorrhage: implications for the role of oxidative stress in cerebral vasospasm. Neurol Res 2009; 31: 195-199.

10 Pasquini A, Luchetti E, Marchetti V, Cardini G, Iorio EL. Analytical performances of d-ROMs test and BAP test in canine plasma. Definition of the normal range in healthy Labrador dogs. Vet Res Commun 2008; 32: 137-143.

11 Cesarone MR, Belcaro G, Carratelli M, Cornelli U, De Sanctis MT, Incandela L, Barsotti A, Terranova R, Nicolaides A. A simple test to monitor oxidative stress. Int Angiol 1999; 18: $127-130$.

12 Trotti R, Carratelli M, Barbieri M. Performance and clinical application of a new, fast method for the detection of hydroperoxides in serum. Panminerva Med 2002; 44: 37-40.

13 Atabek ME, Vatansev $\mathrm{H}$, Erkul I. Oxidative stress in childhood obesity. J Pediatr Endocrinol Metab 2004; 17: 1063-1068.

14 Kamezaki F, Yamashita K, Kubara T, Suzuki Y, Tanaka S, Rkouzuma R, Okazaki M, Tasaki $\mathrm{H}$, Otuji Y. Derivatives of reactive oxygen metabolites correlates with highsensitivity C-reactive protein. J Atheroscler Thromb 2008; 15: 206-212.

15 Sakane N, Fujiwara S, Sano Y, Domichi M, Tsuzaki K, Matsuoka Y, Hamada T, Saiga K, Kotani K. Oxidative stress, inflammation, and atherosclerotic changes in retinal arteries in the Japanese population; results from the Mima study. Endocrine $J$ 2008; 55: 485-488.

16 Hirose $\mathrm{H}$, Kawabe $\mathrm{H}$, Komiya N, Saito I. Relations between serum reactive oxygen metabolites (ROMs) and various inflammatory and metabolic parameters in a Japanese population. J Atheroscler Thromb 2009; 16: 77-82.

17 Lubrano V, Vassalle C, L'abbate A, Zucchelli GC. A new method to evaluate oxidative stress in humans. Immunoanal Biol Spec 2002; 17: 172-175.

18 Hong JY, Sato E, Kira Y, Nishikawa M, Shimada K, Inoue M. Curcuma aromatic inhibits diabetic nephropathy in the rat. J Food Sci 2006; 71: S626-S632.

19 Regano N, lorio EL, Guglielmi A, Mazzuoli S, Francavilla A, Fregnan S, Leogrande G, Guglielmi FW. The assessment of oxidative stress in clinical practice and its importance in nutrition. Nutr Ther Metabol 2008; 26: 149-162.

20 Morishita Y, Hanawa S, Chinda J, Tsunematsu S, Kusano E. Effects of aliskiren on blood pressure and the predictive biomarkers for cardiovascular disease in hemodialysisdependent chronic kidney disease patients with hypertension. Hypertens Res 2011; 34: 308-313.

21 Fukui T, Momoi A, Yasuda T. Attention for the interpretation of measurements of brachial-ankle pulse wave velocity. Ningen Dock 2005; 19: 29-32.

22 Libby P. Inflammation in atherosclerosis. Nature 2002; 420: 868-874.

23 Ridker PM, Cushman M, Stampfer MJ, Tracy RP, Hennekens CH. Inflammation, aspirin, and the risk of cardiovascular disease in apparently healthy men. N Engl J Med 1997; 336: 973-979.

24 Pearson TA, Mensah GA, Alexander RW, Anderson JL, Cannon 3rd RO, Criqui M, Fadl YY, Fortmann SP, Hong Y, Myers GL, Rifai N, Smith Jr SC, Taubert K, Tracy RP, Vinicor F. Markers of inflammation and cardiovascular disease: application to clinical and public health practice: A statement for healthcare professionals from the Centers for Disease Control and Prevention and the American Heart Association. Circulation 2003; 107: 499-511.

25 Fujita K, Nishizawa H, Funahashi T, Shimomura I, Shimabukuro M. Systemic oxidative stress is associated with visceral fat accumulation and the metabolic syndrome. Circ $J$ 2006; 70: 1437-1442.

26 Ihara H, Hashizume N, Hasegawa T, Yoshida M. Antioxidant capacities of ascorbic acid, uric acid, alpha-tocopherol, and bilirubin can be measured in the presence of another antioxidant, serum albumin. J Clin Lab Anal 2004; 18: 45-49.

27 Sohal RS, Orr WC. Relationship between antioxidants, prooxidants, and the aging process. Ann N Y Acad Sci 1992; 21: 663:74-663:84.

28 Mandas A, Congiu MG, Balestrieri C, Mereu A, lorio EL. Nutritional status and oxidative stress in an elderly Sardinian population. Mediterr J Nutr Metab 2008; 1: 99-107. 